\title{
Laparoscopic Repair for Enterocutaneous Fistula Caused by Laparoscopic Right Hemicolectomy for Pan-Peritonitis due to Cecal Cancer Perforation
}

\author{
Min Hong Lee, M.D., Min Gyu Kim, M.D., Ph.D. \\ Department of Surgery, Hanyang University Guri Hospital, Hanyang University School of Medicine, Seoul, Korea
}

Enterocutaneous fistula (ECF) can occur after any intra-abdominal procedure and is a concern for most surgeons. We report a case of laparoscopic treatment of an ECF in a 65-year-old male patient who had undergone a laparoscopic right hemicolectomy for peritonitis due to perforated colon cancer four months previously. Two weeks after discharge from the hospital, he visited the outpatient clinic complaining of a malodorous abscess draining from the right trocar site. Although we allowed two months for the fistula to close naturally, it did not close and formed an abscess. The patient subsequently underwent a laparoscopic colectomy of the fistula between the colonic stump and the skin. The operation time was 110 minutes, and he was discharged 10 days after surgery without any postoperative complication.

Keywords: Enterocutaneous fistula, Laparoscopy, Colectomy, Colon cancer, Bowel perforation
Received May 21, 2020

Revised 1st June 28, 2020

2nd August 14, 2020

Accepted August 20, 2020

Corresponding author

Min Gyu Kim

Department of Surgery, Hanyang

University Guri Hospital, Hanyang

University School of Medicine, 153

Gyeongchun-ro, Guri 11923, Korea

Tel: +82-31-560-2294

Fax: +82-31-566-4409

E-mail: md9650@hanyang.ac.kr

ORCID:

https://orcid.org/0000-0001-9943-0083

Copyright $\odot 2020$ The Journal of Minimally Invasive Surgery. All rights reserved.
This is an Open Access article distributed under the terms of the Creative Commons Attribution Non-Commercial License (http:// creativecommons.org/licenses/by-nc/4.0/) which permits unrestricted non-commercial use, distribution, and reproduction in any medium, provided the original work is properly cited.

\section{INTRODUCTION}

Formation of enterocutaneous fistula (ECF) after surgery is a serious postoperative complication that can occur after any intra-abdominal procedure. Particularly, an intestinal fistula may occur in areas where the bowel wall is damaged or where an anastomosis is performed during surgery. ${ }^{1,2}$ Despite improvements in antibiotics, intensive care, nutrition support, wound care, surgical instruments, and surgical techniques, the mortality rate from ECF remains high $(5.3 \sim 10.8 \%)^{3,4}$

Every surgeon needs to make efforts to minimize the occurrence of ECF and should be knowledgeable about appropriate treatment methods is needed. Previous reports indicate that an
ECF lasting more than 2 months closed spontaneously in less than $10 \%$ of patients. ${ }^{6}$ Therefore, surgical treatment is considered for ECFs persisting longer than 2 months. However, surgical treatment for ECF is highly invasive because of severe intraperitoneal adhesions and direct invasion with surrounding structures. To the best of our knowledge, laparoscopic bowel resection of an ECF that was caused by surgery for peritonitis has been rarely reported. Therefore, we report of a 65 -year-old male who underwent a laparoscopic colectomy of a fistula between the colonic stump and the trocar site. 


\section{CASE REPORT}

A 65-year-old man was referred to OOO Hospital for treatment of perforated colon cancer. He had visited a local clinic one week previously because of right lower abdominal pain. One day before transferring to our hospital for surgical treatment, he had visited another hospital and was diagnosed with pan-peritonitis caused by cecal cancer perforation. The patient showed severe abdominal pain accompanied by abdominal tenderness and rigidity due to pan-peritonitis.

Although the patient had no specific underlying diseases, the ASA (American Society of Anesthesiologist) score was $3 \mathrm{E}$ for old age and heavy smoker status (40 pack-year history). The patient's BMI (Body Mass Index) was $19.6 \mathrm{~kg} / \mathrm{m}^{2}$ (height, $158 \mathrm{~cm}$; weight $49 \mathrm{~kg}$ ). Preoperative blood tests revealed no specific findings other than increased leukocytes and C-reactive protein. Abdominal computed tomography showed a $6 \mathrm{~cm}$-sized, polypoid mass with pericolic infiltration and several pericolic lymphadenopathy (Fig. 1A).

The patient's first radical right hemicolectomy was performed by a totally laparoscopic method using an intracorporeal anastomosis. The patient position was adjusted to a 10- to 20-degree tilt with the left side down and slight Trendelenburg. After the initial trocar was placed and the dissection began, the ileocolic pedicle was identified by gently placing the ileocecal junction from the retroperitoneum toward the right lower area. Laparoscopic suction was performed for contamination and possibility of cancer cell spillage from perforation. The perforation size was minimzed by using a 2-0 black silk suturing to prevent cancer cell spillage during surgery. Complete dissection in this bloodless plane was performed laterally over Gerota's fascia to the abdominal wall laterally and cranially over the duodenum and head of the pancreas entering the lesser sac. Once the ileocolic artery was isolated, it was ligated with surgical clips.

After dividing the ileal and lateral colonic attachments, an incision was made where the peritoneal reflection meets the lateral wall of the cecum. After completing a dissection around the hepatic flexure, the hepatocolonic ligament was divided. After mobilization of the ileum and colon, the right branches of the middle colic vessels were ligated by surgical clips.

The proximal ileal and distal colonic stumps were made preferentially using laparoscopic linear staplers (ECHELON FLEXTM ENDOPATH $^{\circledR}$ Stapler 60 ). Specimen extraction was conducted through the umbilical port by extending the incision (Fig. 1B). The ulceroinfiltrating mass with perforation is noted at cecum to ileocecal valve, measurig $6.5 \times 4.5 \mathrm{~cm}$ in dimensions. After reformation of the pneumoperitoneum, a side-to-side anastomosis between the colon and the ileum was performed using laparoscopic linear staplers (Fig. 2). The operation time from skin incision to skin closure was 180 minutes. An anesthesiologist calculated the estimated blood loss (EBL) as $200 \mathrm{cc}$. On the sixth postoperative day, the patient started a soft diet, and he was discharged from the hospital on the 11th postoperative day. There was no postoperative complication during the hospital stay. Histologically, glands of moderately differentiated adenocarcinoma were invaded into the pericolic soft tissue without nodal involvement (0/20). Proximal and distal resection margin showed 12, 15 $\mathrm{cm}$, respectively. According to the tumor-node-metastasis (8th AJCC TNM) staging system, the staging was T3N0M0, Stage IIA.

Two weeks later, the patient presented to the outpatient clinic and complained of an abscess with an odor at the troca insertion site. Despite two months of wound care for the abscess, the

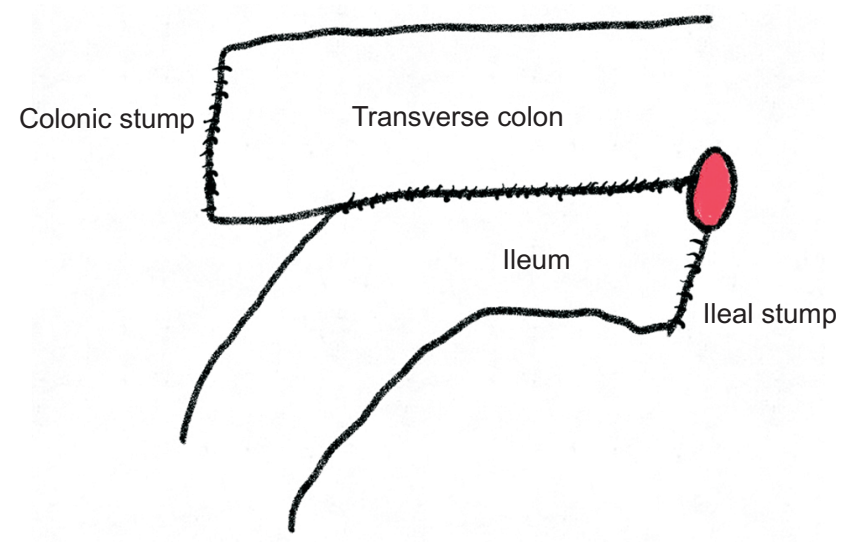

Fig. 2. Linear stapler was inserted into red circle to perform side-to-side anastomosis (overlap method).
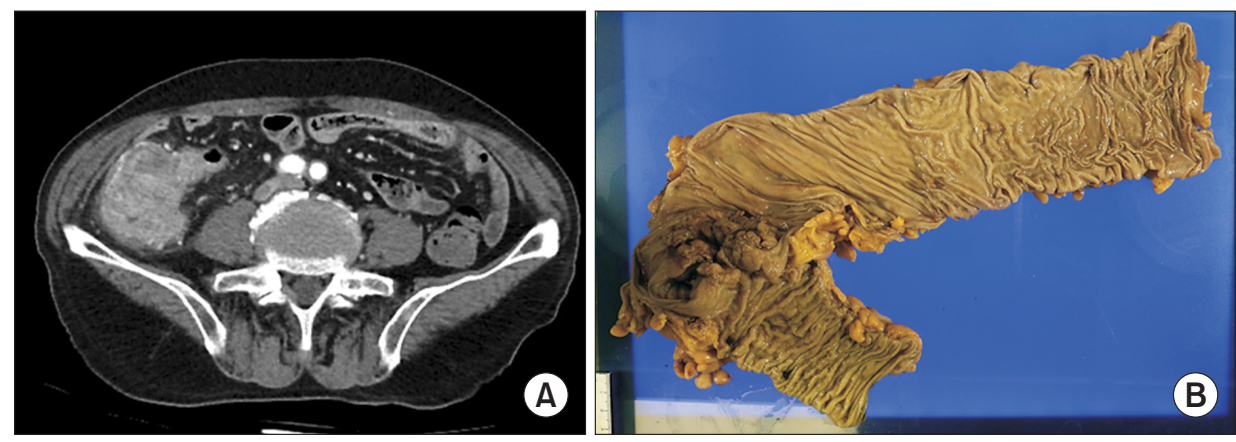

Fig. 1. (A) Abdominal computed tomography showed mass-like lesion with pericolic infiltration and edematous change. (B) A $6.5 \mathrm{~cm}$ tumor was located in the cecum. 
wound discharge did not improve, and fistulography showed a communication between the skin at the trocar site and the colon (Fig. 3). The patient refused to wait any longer for natural closure of the tract and wanted surgical treatment, so we planned for laparoscopic surgery.
After laparoscopically entering the abdomen and creating a pneumoperitoneum, we lysed the peritoneal adhesions in the right abdominal cavity. Next, the fistula between the right trocar insertion site and the colon stump site was confirmed (Fig. 4A). After separating the fistula from the abdominal wall (Fig. 4B),
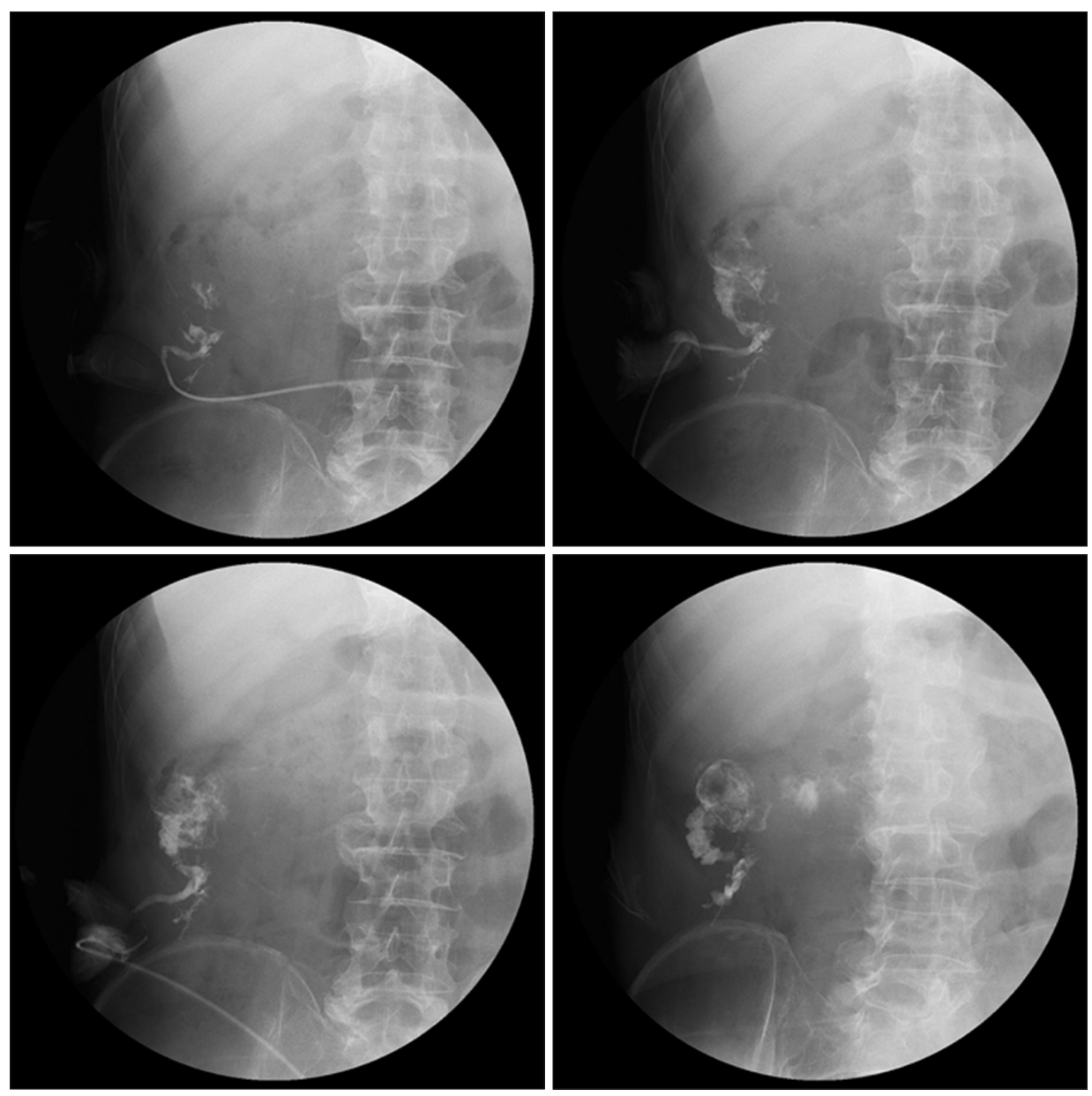

Fig. 3. Fistulography showed a communication between the skin and bowel loop.
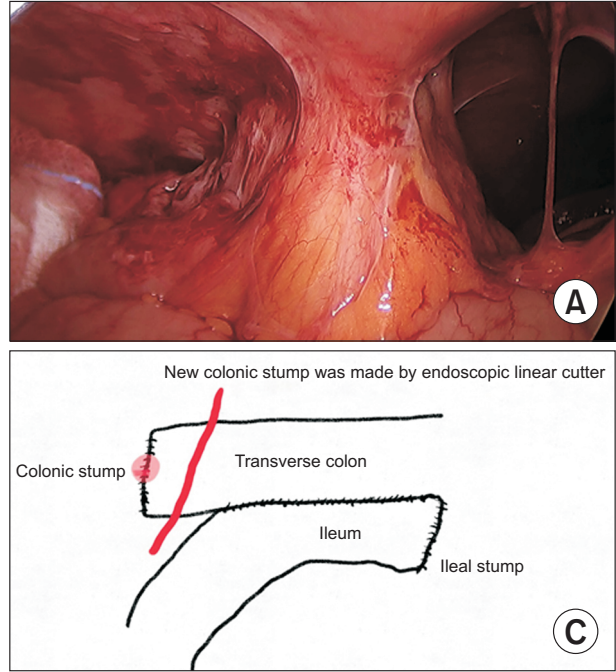
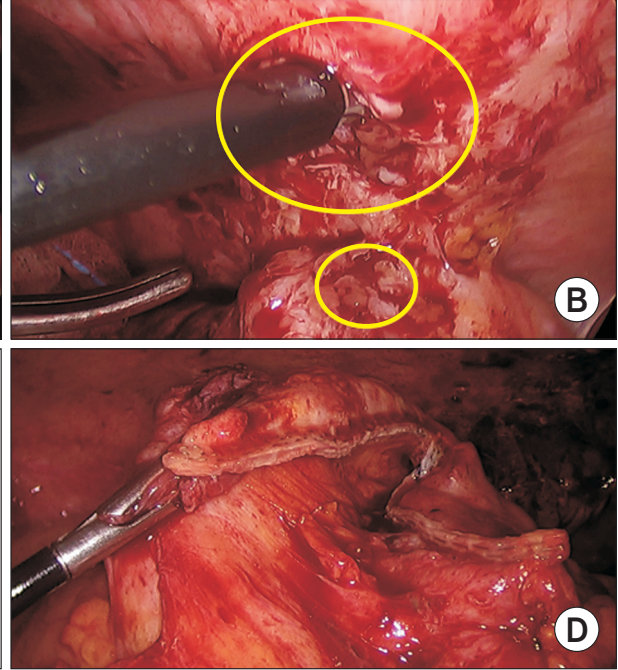

Fig. 4. (A) Enterocutaneous fistula (ECF) can be confirmed by laparoscpy. (B) the ECF was separated from the abdominal wall. (C, D) Colectomy including fistula was performed. 
the colon stump and the fistula were mobilized. The distal colonic stump was rebuilt using a laparoscopic linear stapler across the anastomosis (Fig. 4C, D). Finally, the specimen was extracted through the umbilical port site. The operation time was 110 minutes (from skin to skin), and most of the time was spent on adhesiolysis. An anesthesiologist calculated the EBL as $100 \mathrm{cc}$. On the fifth postoperative day, the patient started a soft diet, and he was discharged from the hospital on the 10th postoperative day. There was no postoperative complication during the recovery stay. Eighteen months later, he continues to be doing well without surgery-related complications.

Approval (GURI 2020-06-009) was obtained from the institutional review board of Hanyang University Guri Hospital of Korea, College of Medicine.

\section{DISCUSSION}

Development of an ECF confers high morbidity and mortality despite advances in surgical instruments, surgical techniques, nutrition support, and intensive care. ${ }^{3-5}$ An ECF can be caused by surgery, inflammatory bowel disease, diverticulitis, trauma, and malignancy. More than $75 \%$ of cases of ECF are associated with surgery, and an anastomotic leak following bowel resection is known to be an important cause. ${ }^{1,2}$ In our case, we believed that the formation of ECF was caused by a sustained inflammatory response from minor leak or wound infection. The ileocolic anastomosis showed a leak rate of $0.02 \sim 4 \%{ }^{7}$ In addition to technical problems, preoperative factors related to the patient's comorbidity or general condition can also cause leakage.

When an ECF develops, conservative treatment is considered the priority until the fistula naturally closes. The main focus of conservative treatment is early recognition, wound care, nutrition support, treatment of sepsis, and correction of electrolyte imbalance. ${ }^{7}$ If conservative treatment fails, surgical treatment should be planned.

The surgical treatment of an ECF is a time consuming and technically difficult operation, with a high possibility of postoperative complications. It is well known that adhesions are an important risk factor for postoperative complications. The operation should be delayed for several months to decrease the degree of adhesion. ${ }^{4}$ Also, because $30 \%$ of recurrent fistulas are caused by bowel injury that occurred during ECF surgery, gentle traction and meticulous dissection are needed to avoid bowel injury during ECF surgery. ${ }^{8}$ Moreover, the surgery should be aimed at bowel resection including the fistula tract because of the high recurrence rate due to oversewing or wedge resection.

Adhesion occurs in most patients who undergo an intraabdominal surgery. The risk factors for complications caused by adhesions are type of surgery (laparoscopy or open surgery), number of previous laparotomies, damage to the peritoneum, and intra-operative complications during the surgery., ${ }^{9,10}$ In particular, bleeding and intra-abdominal infection increase adhesion formation.

Because of these risk factors, our surgical team considered the pros and cons of laparoscopic surgery in the planning and preparation phase for reoperation in the present case. Because the first surgery was laparoscopic, we decided that laparoscopy would be sufficient as a method of reoperation. During the second surgery, we were convinced that the first laparoscopic surgery minimized the degree of adhesion from the peritonitis secondary to bowel perforation. We believed that laparoscopic surgery could be beneficial for securing an operational field of view and more meticulous dissection.

However, not all cases can benefit from laparoscopic surgery. Successful completion of the learning period required for laparoscopic surgery is a critical prerequisite for better surgical outcomes; certainly, only experts in laparoscopic surgery should try laparoscopic surgery. Also, we believed that laparoscopic surgery is could be tried if the most part of abdomen is soft, except around the ECF area, regardless of past history of laparoscopic or open surgery. Especially for planning of ECF surgery, it seems appropriate to avoid the acute phase because of severe inflammation. Therefore, the purpose of the present report was to share our treatment experience of a patient who underwent an initial laparoscopic surgery for peritonitis and a second laparoscopic surgery for subsequent occurrence of an ECF.

\section{ORCID}

Min Hong Lee, https://orcid.org/0000-0001-8054-9308

Min Gyu Kim, https://orcid.org/0000-0001-9943-0083

\section{AUTHORS' CONTRIBUTIONS}

Conceptualization: Min Gyu Kim. Formal analysis: Min Gyu Kim. Methodology: Min Gyu Kim. Writing-original draft: Min Hong Lee. Writing-review and editing: Min Gyu Kim.

\section{CONFLICT OF INTEREST}

None.

\section{FUNDING}

None.

\section{ACKNOWLEDGMENTS}

None. 


\section{REFERENCES}

1) Berry SM, Fischer JE. Classification and pathophysiology of enterocutaneous fistulas. Surg Clin North Am 1996;76:1009-1018.

2) Fischer JE. The pathophysiology of enterocutaneous fistulas. World J Surg 1983;7:446-450.

3) Hollington P, Mawdsley J, Lim W, Gabe SM, Forbes A, Windsor AJ. An 11-year experience of enterocutaneous fistula. Br J Surg 2004;91:1646-1651.

4) McIntyre PB, Ritchie JK, Hawley PR, Bartram CI, Lennard-Jones JE. Management of enterocutaneous fistulas: a review of 132 cases. Br J Surg 1984;71:293-296.

5) Draus JM, Jr., Huss SA, Harty NJ, Cheadle WG, Larson GM. Enterocutaneous fistula: are treatments improving? Surgery 2006;140:570576; discussion 576-578.
6) Evenson AR, Fischer JE. Current management of enterocutaneous fistula. J Gastrointest Surg 2006;10:455-464.

7) Phitayakorn R, Delaney CP, Reynolds HL, et al. Standardized algorithms for management of anastomotic leaks and related abdominal and pelvic abscesses after colorectal surgery. World J Surg 2008;32:1147-1156.

8) Lynch AC, Delaney CP, Senagore AJ, Connor JT, Remzi FH, Fazio VW. Clinical outcome and factors predictive of recurrence after enterocutaneous fistula surgery. Ann Surg 2004;240:825-831.

9) Gutt CN, Oniu T, Schemmer P, Mehrabi A, Büchler MW. Fewer adhesions induced by laparoscopic surgery? Surg Endosc 2004;18:898906.

10) Levrant SG, Bieber E, Barnes R. Risk of Anterior Abdominal Wall Adhesions Increases with Number and Type of Previous Laparotomy. J Am Assoc Gynecol Laparosc 1994;1:S19. 